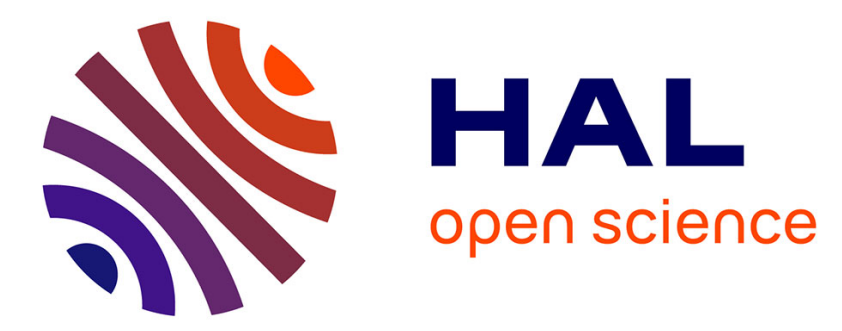

\title{
Musical aero-acoustics of the clarinet
}

A. Hirschberg, Joel Gilbert, A. Wijnands, A. Valkering

\section{To cite this version:}

A. Hirschberg, Joel Gilbert, A. Wijnands, A. Valkering. Musical aero-acoustics of the clarinet. Journal de Physique IV Proceedings, 1994, 04 (C5), pp.C5-559-C5-568. 10.1051/jp4:19945120 . jpa-00252796

\section{HAL Id: jpa-00252796 https://hal.science/jpa-00252796}

Submitted on 1 Jan 1994

HAL is a multi-disciplinary open access archive for the deposit and dissemination of scientific research documents, whether they are published or not. The documents may come from teaching and research institutions in France or abroad, or from public or private research centers.
L'archive ouverte pluridisciplinaire HAL, est destinée au dépôt et à la diffusion de documents scientifiques de niveau recherche, publiés ou non, émanant des établissements d'enseignement et de recherche français ou étrangers, des laboratoires publics ou privés. 


\title{
Musical aero-acoustics of the clarinet
}

\section{A. HIRSCHBERG, J. GILBERT* ${ }^{*}$ A.P.J. WIJNANDS and A.M.C. VALKERING}

Vakgroep Transportfysica, Eindhoven Univ. of Technology, P.O. Box 513, 5600 MB Eindhoven, The Netherlands

* LAUM, URA 1101 du CNRS, Univ. du Maine, Av. O. Messiaen, BP. 535, 72017 Le Mans, France

\begin{abstract}
We present a review of literature on aspects of sound production in a clarinet which involve non-linearities of the flow in the instrument. We discuss the flow in the reed channel, the contribution of turbulence to the sound production, the vortex shedding at tone holes and the pipe termination and finally we discuss the non-linear wave deformation in the pipe. Most of these effects can only be described qualitatively on the basis of existing theories and the experimental data are insufficient. Further research is welcome.
\end{abstract}

Bouasse dixit: J'ai appris par les affiches que la Faculté des Sciences de Toulouse possède un Institut de Mécanique des fluides; est-il besoin de dire que je n'ai rien de commun avec cet institut, pas davantage avec l'Institut Electrotechnique où cette mécanique est également en honneur. Je préviens le lecteur pour qu'il ne soit pas surpris des contradictions entre ce que je signe et ce qu'élabore ces messieurs.[1].

1. Musical-acoustics, Fluid dynamics and Aero-acoustics Acoustics, as the study of linear wave propagation in fluids, is an approximation of fluid dynamics. Aero-acoustics is the study of the coupling between a region in space $^{1}$ where the acoustical approximation prevails and flow regions where non-linear phenomena are essential and responsible for the transfer of energy to the acoustical flow.

In the past decade a strong activity in the field of the musical acoustics of wind instruments has been developed in France. The research carried out in close contact with wind instrument manufacturers, both individuals and industrials, has focussed (in first instance) on the relationship between linear acoustical properties of pipes expressed in terms of impedances and the quality of the instruments. Practical tools, like the software Resonans[2], are now available and are used in the design of new instruments. The research in this important field of musical acoustics is still going on.

A following challenge was the development of numerical sound synthesizers based on a physical modelling as proposed in the paper of McIntyre e.a.[3]. For single reed instruments: the Clarinet and the Saxophone, pioneer work was performed at IRCAM by Ducasse[4]. The quality of the sound produced indicates that musically interesting synthesizers can be developed on the basis of simple physical models. The work was followed by attempts to obtain models for the flue organ pipe which appeared to be less satisfactory. Fundamental research on the fluid dynamics of wind instruments was clearly needed in that case. In collaboration with the laboratory for Fluid Dynamics and Heat Transfer of TUE, the Laboratory of Musical Acoustics of Paris VI [5] and the IRCAM [6] have been working on the fluid mechanics of flue instruments and seem to have changed drastically the basic ideas in this field of research. The currently accepted models described in the recent book of Fletcher

\footnotetext{
${ }^{1}$ In which the listener is immerged.
} 
and Rossing[7] appear to be oversimplified from a fluid dynamic point of view. The classical model overestimates stationary pressure fluctuations in the pipe by more than an order of magnitude. The key of this deficiency appears to be the local vortex shedding at the labium which was already pinpointed by Howe[8] as an important aero-acoustical feature in the flute. This vortex shedding, which was assumed to be a second order effect by Fletcher[9], is not only responsible for $90 \%$ of the energy losses of the fundamental mode of flue organ pipes, it also largely determines the amplitude of higher harmonics.

A similar situation appeared in human voiced sound production. The original two mass models of vocal folds of Ishizaka and Matsudaira[10] was mainly improved by an increasing complexity of the mechanical model. As shown by Pelorson[11] a significant improvement of the glottal signal produced by simple two mass models can be obtained by a less caricatural fluid dynamics model and by a model in which the mechanical contact of the folds is "disconnected" from the flow interruption ${ }^{2}$.

These examples clearly demonstrate that an integrated approach in which the acoustic field and the flow are incorporated in a single musical aero-acoustic model is useful. In the present paper we discuss the relevance of such an approach for the synthesis of clarinet sound and the design of instruments. As the existing models of a clarinet do already provide a reasonable sound, we ask the question: what aspects of the clarinet behaviour are not yet fully understood because of the lack of a systematic "musical aero-acoustic" approach?

2. Volume flow control by the reed oscillation The most obvious non-linear fluid dynamical phenomenon in a clarinet is the modulation of the volume flow through the reed channel by the oscillation of the reed. In basic models, the modulated volume flow at the entrance of the pipe acts as an oscillating piston which generates the acoustic waves in the pipe: it is the sound source. At low frequencies $\left(f_{0}\right)$ of the fundamental and reasonable mouth blowing over-pressure $\Delta p_{m}$, the flow in the reed channel can be considered to be frictionless, incompressible and quasi-stationary[12]. The action of friction however is essential to explain the flow control mechanism. Friction is responsible for the separation of the flow from the walls in the reed channel which results into the formation of a free jet with a height $h_{j}$ closely related to the reed channel height $h_{r}$. The intrinsic instability of the free jet results into a chaotic vortical motion called turbulence. Typical for a turbulent flow is that even at high Reynolds numbers $R e_{h}=\frac{U_{B} h_{j}}{\nu}$ the kinetic energy of the jet is very efficiently dissipated by energy transfer to small vortical structures ${ }^{3}$. As the ratio of typical jet height $h_{j}$ to acoustic pipe diameter $D$ is very small $\left(h_{j} / D\right)=O\left(10^{-1}\right)$, we can neglect the recovery of total pressure in the mouthpiece.

As a consequence in first approximation the pressure $\mathrm{p}(0)$ in the mouthpiece is uniform. This yields a volume flux $\Phi$ through the reed channel given by:

$$
\Phi=W h_{j} \sqrt{\frac{2\left[p_{m}-p(0)\right]}{\rho_{0}}}
$$

where $W$ is the (effective) width ${ }^{4}$ of the reed channel and $p(0)$ is the pressure at the entrance of the pipe. The reed channel consists of two parts: a)the front slit delimited by the edge of the mouthpiece tip (the baffle) and the reed and b) the lateral slits between the lay (curved wall against which the reed is pressed) and the reed. We will further assume that the reed channel geometry is determined by the front reed channel height $h_{r}$ at the tip of the reed. In first approximation the

\footnotetext{
${ }^{2}$ As the two mass model is a two dimensional representation of a three dimensional movement it seems reasonable to assume a mechanical contact before the flow is stopped. We assume a mechanical contact at a critical glottal apperture, before the glottis closes completely. This idea can also be used in musical acoustics to describe the oscillation of the player's lips in brass instruments.

${ }^{3} U_{B}=\sqrt{\frac{2 \Delta p_{m}}{\rho_{0}}}$ is the Bernoulli velocity, $\nu$ is the kinematic viscosity of air, $\Delta p_{m}=p_{m}-p_{a}$ is the over-pressure (above the athmospheric pressure $p_{a}$ ) in the player's mouth and $\rho_{0}$ is the mean density of air

${ }^{4}$ Typically $W$ is of the order of the pipe diameter $D$.
} 
front reed channel height is uniform. The variation in the height of the lateral reed channels can be incorporated into a functional relationship between $W$ and $h_{r}$. As suggested in the literature [7] this may imply a non-linear relationship between $\Phi$ and $h_{r}$. This relationship is not expected to be universal, it depends on the reed properties and the lay geometry. Typically the reed channel length $L_{r}$ is of the order of one millimeter.

A considerable research effort has been carried out in parallel at the LAUM and at the TUE to obtain a reliable model for the relationship between the jet height $h_{j}$ and the reed channel height $h_{r}$. Using simplified geometries van Zon e.a.[13] demonstrated that in steady flow conditions two types of reed channel flow could exist. A fully separated jet flow for short $\left(L_{r} / h_{r}<4\right)$ reed channel length $L_{r}$. In this case, due separation at the sharp edge of the reed and the subsequent vena-contracta effect the relationship $h_{j}=\alpha h_{r}$ prevails where $\alpha<1$ is called the vena-contracta factor. As expected from potential flow theory van Zon found: $\alpha \approx 0.5$. For long reed channels $\left(L_{r} / h_{r}>4\right)$ the viscous entrainment by the jet in the reed channel induces a reattachment of the flow to the walls. At high Reynolds numbers $\left(\operatorname{Re}_{h}\left(\frac{h_{r}}{L_{r}}\right)>10^{2}\right)$ this implies $\alpha \approx 1$. At lower Reynolds numbers an analytical model proposed by van Zon[13 ${ }^{5}$ describes the transition towards a Poiseuille flow and predicts within $5 \%$ the experimental data available. Data obtained at LAUM [15] confirmed these results and provide additional information on the influence of the rounding off of the edges of the reed channel entrance and the confinement of the mouthpiece in the player's mouth. These preliminary results obtained in a highly simplified model of the player's mouth show a drastic increase of $\alpha$ from an initial value of 0.61 up to a value of 0.85 .

Measurements of stationary flow in actual saxophone mouthpieces by Maurin[15] and clarinet mouthpieces by Valkering[16] seem to agree with the measurements in simplified geometries. They do not provide a significant improvement of our understanding because of the uncertainty in the geometry of the reed channel. In particular the effective reed channel width $W$ is difficult to estimate (there is a large uncertainty in the contribution of the lateral slits). We have to confess that we did not find any significant correlation between the musical quality of different mouthpieces and our stationary flow measurements. This is not only due to the uncertainties in the reed channel geometry. We suspect that unsteadiness of the flow is an essential feature, so that stationary measurements have a limited value. Gilbert[17], using the set-up designed by Meynial[18], was not able in dynamical measurements of the flux $\Phi$ to observe a transition between the fully separated jet flow regime and the reattached flow regime. The data of Gilbert[17] indicate that the best we can do in a simplified theory is to assume a constant vena contracta factor $\alpha$. Unfortunately the dynamic data of Gilbert[17] are unreliable for $L_{r} / h_{r}>10$. As we expect in analogy with voiced sound production that the final phase of reed closing $\left(h_{r} \rightarrow 0\right)$ is crucial for the generation of higher harmonics, this lack of reliable data is a problem. We may conclude that unless better dynamical data are available one should not use a complex model. This would justify the type of reed flow equations proposed by Fletcher and Rossing[7] using equation (1) with modified exponents for $h_{j}$ and $\left(p_{m}-p(0)\right)$. We should realize that these exponents do not have any simple physical meaning nor universal value. They are fit parameters only.

3. The Bernoulli force on the reed As the Bernoulli equation associates an acceleration of the fluid with a decrease of pressure, it has become an accepted idea in musical acoustics literature that a flow through a reed would always imply a net hydrodynamic force tending to close the reed channel which is referred to as the Bernoulli force $F_{B}$. As explained by Hirschberg([12],[14]) the Bernoulli force can exist in a clarinet mouthpiece either as a result of flow separation at the channel entrance followed by reattachment or as a result of delayed flow separation at the reed channel exit $\left(h_{r}<h_{j}\right)$. In the first case the Bernoulli force increases with increasing reed channel height. In the second case the Bernoulli force depends on the ratio minimum reed channel height and the jet height $h_{j}$. This second case corresponds to the Bernoulli force in the glottis and may increase with

\footnotetext{
${ }^{5}$ See correction of typing errors, in formulas of the original publication[13], in the NAG paper of Hirschberg[14]. The factors $(1-\delta)$ should be replaced by $(1-\delta)^{2}$.
} 
decreasing $h_{r}$ (but this is not obvious as often assumed in the literature).

A Bernoulli force $F_{B}\left(h_{r}\right)$ which depends only on the reed channel aperture $h_{r}$ is not able, under steady oscillation conditions, to transfer energy from the flow to the reed oscillation because([19][20]) for a periodic reed movement: $\oint F_{B} d h_{r}=0$. An energy transfer from the flow to the reed, through $F_{B}$, can be due to a modulation of the Bernoulli force by: a) Acoustical fluctuation of the mouthpiece pressure due to pipe resonance, b) Difference in the reed channel geometry between the opening and closing phase, such as obtained by the two mass model of the vocal folds oscillation(more than one mechanical degree of freedom [10]), c) Inertia of the reed flow (harmonium reed[21]) or d) A time dependence or non-linear behaviour of the separation/reattachment phenomenon in the reed channel ${ }^{6}$. The analogy between the reattachment (by viscous entrainent) and the Coanda effect led [12] to the idea that this phenomenon, corresponding to the two flow regimes observed by van Zon[13], could be responsible for reed oscillation in absence of acoustical feedback for a simple reed (one mechanical degree of freedom). Attempts to demonstrate experimentally this phenomenon on a valve with a single mechanical degree of freedom, reported by Hirschberg[14], are illustrated in Figure 1.

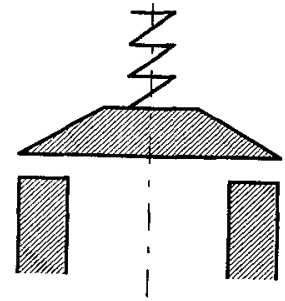

a)

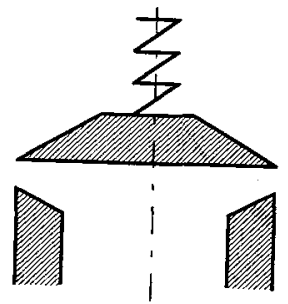

b)

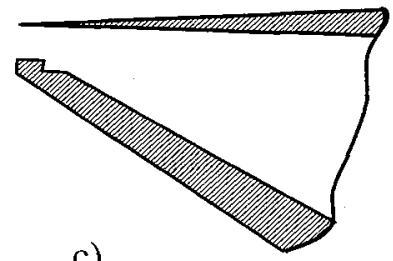

c)

Figure 1: Influence of the reed channel geometry on the reed oscillation: a) non-oscillating simple valve, b) oscillating simple valve and c) reed channel for easy emmission.

When the valve had a uniform "reed" channel height $h_{r}$, self sustained oscillations, could not be obtained for the typical Strouhal numbers $S r_{h}=f_{0} L_{r} / U_{B}=O\left(10^{-3}\right)$, based on the resonance frequency of the mass spring system $f_{0}$ and the reed channel length $L_{r}$. However when the "reed" channel was made divergent under an angle of $30^{\circ}$, the valve did oscillate. This dramatic effect of the geometry of the reed channel is confirmed by the experience of Benade[22], that an apparently minor modification (rounding off) of the edges of the reed channel exit strongly influences the musical sound produced by a saxophone. This is also the experience of mouthpiece manufacturers (J. Minor, private communication(1991)): after the curvature of the lay, the geometry of the baffle is the most critical part of mouthpiece design. Not only the spectral distribution of the sound is influenced but also the emmission facility is affected. When a sharp edged "ditch" is carved just downstreet of the reed channel exit (Figure 1), one can experience an easier attack transient (D.Bechert, private communication(1992)). We therefore conclude that the details of the flow in the reed channel are crucial and that the most important effect is the movement of the separation point at the flue channel exit. The fascinating results obtained by Pelorson[11] with a quasi-stationary flow separation model in a glottis, indicate that calculations based on simplified boundary layer theory can provide some insight into the relationship between the geometry of the reed channel and the musical behaviour of a mouthpiece. One could also suspect the possibility of a strong Coanda effect at the reed channel exit. As noted by Pelorson[11], in the case of vocal folds, such a Coanda effect is strongly affected

\footnotetext{
${ }^{6}$ The possibility of a hysteresis in a separation/reattachment phenomenon corresponds to our common experience of the Coanda effect: Once tea starts flowing along the wall of the tea-pot, we have to do a considerable effort to restore the separated jet flow.
} 
by the unsteadiness of the flow. It might therefore be essential to study the mouthpiece behaviour in unsteady flow experiments or in unsteady numerical flow simulations.

4. Turbulence and chaos The addition of noise to synthetic sound is necessary to obtain a reasonable naturalness. In speech research this problem has already been recognized, and models of turbulent noise production have been proposed [23]. The introduction of this aspect to musical sound synthesis (based on physical models) is only recent ([24],[25]). It is important to realize that this is more than a simple addition of "noise" to the produced sound. As musical instruments are non-linear oscillator they exhibit chaotic behaviour. A certain level of non-periodicity is an essential characteristic of musical sound [26] and some musicians use multiphonics involving chaotic behaviour [27]. The turbulence as a chaotic fluid motion can therefore play a double role: a) produce "noise" in the sound reaching the listener and b) trigger bifurcations in the oscillation. Recent data analysis studies such as performed by [28] are an important source of information on this phenomenon because modelling of turbulence or local flow measurements are unreliable. Turbulence is an important detail in a clarinet. Finnaly we note that, turbulence does not only occur in the jet formed by flow separation at the reed channel exit. Vortex shedding at tone holes or the pipe end can also induce turbulence ${ }^{7}$ (see next section). Furthermore at fortissimo levels the acoustical boundary layers in the pipe could become turbulent ${ }^{8}$.

5. Vortex shedding at tone holes and pipe end Flow separation from walls occurs in a wind instrument, when the acoustical displacement of air particles $\left|u_{a}\right| / \omega_{0}$ is ${ }^{9}$ comparable or larger than the radius of curvature $R$ of edges of tone holes or the pipe termination. The shear layers formed by this flow separation are unstable and roll up into vortices. The interaction between vortices and acoustical flow has already been studied extensively by Bouasse[1]. A recent formal theoretical basis has been supplied for internal flow by Howe[8]. Quantitative experimental information and quasi-stationary models for flow separation at a sharp edged orifice have been discussed by Ingard and Ising[29], Anderson[30] and Cummings[31]. These studies give information on what we can expect at tone holes with sharp edges. In the case of an open pipe end one can find quantitative experimental information and reviews of existing theories in the papers of Disselhorst and van Wijngaarden[32], Cummings and Eversman [33], and Peters e.a.[34],[35]. The paper of Disselhorst and van Wijngaarden provides us with a most useful graph from which we can easily check the importance of vortex shedding in musical instruments in absence of main flow. The impact of main flow has been studied mainly at low acoustical amplitudes $\left|u_{a}\right| / U_{B} \ll 1$, while in musical wind instruments we expect that $\frac{\left|u_{a}\right| D}{U_{B} h_{r}}=O(1)$. There is a lack of information in this field. In particular when we consider a pipe termination with a horn spectacular non-linear effects may occur. A horn can induce whistling analogous to human whistling [35],[36], [37], [38]. Under such conditions strong non-linear behaviour was observed in reflection coefficient measurements by Hirschberg[19].

Another hint of a complex interaction between main flow and vortex shedding at tone holes in a clarinet is the experiment proposed by Benade and reported by Keefe[39]: two clarinets with equal acoustical impedance but thin or thick walls have drastically different properties. Following Keefe[39], and Dane(Private communication(1992)), the thin walled clarinet cannot be played with a typical mouthpiece ${ }^{10}$.

As we expect Strouhal numbers $S r_{D}=f_{0} D /\left|u_{a}\right|<0.3$ upon fortissimo playing in the lower register of a clarinet we do expect strong vortex shedding. Indeed, if we drive a cylindrical pipe with sharp edged end with a clarinet mouthpiece one can observe by flow visualization the spectacular

\footnotetext{
${ }^{7}$ In the literature vortex shedding is some time called turbulence. Vortex shedding does however not automatically imply a chaotic behaviour and should be distinguished from turbulence.

${ }^{8}$ This possibility which has never been considered would imply a considerable modification of friction losses in the pipe

${ }^{9} u_{a}$ is the flow velocity due to the acoustic waves and $\omega=2 \pi f$.

${ }^{10}$ As noted by Nederveen (private communication(1993)) the pipes can both be played by replacing the regular mouthpiece by that of a bass clarinet.
} 
periodic vortex shedding (see [14], [19]). The presence of a horn can strongly reduce such vortex shedding, we can expect flow separation to occur only during the acoustical outflow. As we expect that the acoustic field is fairly described by a standing wave built out of two travelling waves of amplitude $p^{\prime}= \pm \Delta p_{m} / 2$ and associated with a particle velocity $u^{\prime}=\Delta p_{m} / 2 \rho_{0} c_{0}$, the acoustical velocity at the open end is given by: $\left|u_{a}\right|=\Delta p_{m} / \rho_{0} c_{0}$. If we assume a quasi-stationary incompressible behaviour in the horn, we have a pressure $\Delta p=p(L)-p_{a}=-\frac{1}{2} \rho_{0}\left|u_{a}\right|^{2}$ during the acoustical inflow and $\Delta p=p(L)-p_{a}=0$ upon outflow. This corresponds to a relative acoustical energy loss $\Delta E_{a} / E_{a}$, upon reflection of an harmonic wave with an amplitude $\Delta p_{m} / 2$, of:

$$
\frac{\Delta E_{a}}{E_{a}}=\frac{2 \pi f_{0} \int_{0}^{1 / f_{0}} u_{a} \Delta p d t}{\frac{1}{2 \rho_{0} c_{0}}\left(\frac{\Delta p_{m}}{2}\right)^{2}}=\frac{8 \Delta p_{m}}{3 \pi \rho_{0} c_{0}^{2}}
$$

At fortissimo level $\Delta p_{m} / \rho_{0} c_{0}^{2}$ reaches values of $6 \%$, which implies energy losses of $5 \%$ upon reflection of the acoustic wave at the pipe termination! This is comparable to the friction losses which we expect in the pipe of a clarinet $\left(f_{0}=148.5 \mathrm{~Hz}, \mathrm{D} 3\right)$. We do therefore expect that at fortissimo levels flow separation is musically important. If we remove the horn the energy losses by vortex shedding are increased by about a factor two ([32],[35]). The horn should therefore not only be designed on the basis of acoustical considerations, but also to reduce flow separation losses. Except for the simple quasi-stationary model $([29],[40])$ which we have used here, we do not have a well established theory, valid at arbitrary Strouhal numbers. In order to obtain some quantitative information on the non-linearity of the transfer function of acoustical signals from the mouthpiece region to a listener (at $2 \mathrm{~m}$ from the instrument) we therefore carried out some measurements. Typical results of the measurements for the lowest (D3) note of a clarinet, for which we expect the most spectacular effects, is shown in Figure 2. We see that only the fundamental and the third harmonic $\left(f_{3}=4 f_{0}\right)$ do show a significant but modest non-linearity. For the fundamental the transfer function will remain linear as long as the quality factor of the resonator is much larger than unity (D.Ronneberger, private communication (1993)). We expect however that the transfer of energy from the fundamental harmonic to higher harmonics implies a strong non-linear distortion ( [19] and [31]). We are surprised by the fact that only the third harmonic is affected, and only by a few $\mathrm{dB}$. This seems almost negligible compared to what we expected from the vortical power losses estimated above. Similar measurements carried out by Beauchamp[41] on brass instruments seem to indicate a stronger non-linearity than what we observe here. As suggested by Beauchamp[41] this could be due to shock wave formation in brass instruments.

6. Non-linear wave propagation and shock waves $W e$ consider ${ }^{11}$ a sound wave propagating into a uniform quiescent fluid region, a "simple wave". Due to the temperature dependency of the speed of sound $c$ and the variation in particle velocity $u_{a}$ in a sound wave, the high pressure regions in a simple wave move faster ${ }^{12}$ than the low pressure regions. This induces a steepening of the compression side of the wave and a smoothing of the expansion side of the wave. The deformation of a simple wave which is harmonic at $x=0$ is in first approximation ([42],[43]) quantified by the ratio of the amplitude of the fundamental $A_{0}$ and of the first $\left(f_{1}=2 f_{0}\right)$ harmonic $A_{1}$ generated by the deformation:

$$
\frac{A_{1}}{A_{0}}=x \frac{(\gamma+1) 2 \pi f_{0}}{\gamma c_{0}} \frac{A_{0}}{p_{a}}=\frac{x}{2 x_{s}}
$$

where $\gamma=1.4$ is the Poisson constant of air and $p_{a}$ is the atmospheric pressure. When the wave has travelled a distance $x_{s}=\frac{\gamma c_{0}}{(\gamma+1) \pi f_{0}}\left(\frac{p_{a}}{A_{0}}\right)$ the steepening reaches a saturation and a shock discontinuity

\footnotetext{
${ }^{11}$ For simplicity

${ }^{12}$ The wave propagates along a line in the $(\mathrm{x}, \mathrm{t})$ plane given by: $\frac{d x}{d t}=\left(c+u_{a}\right)$. Both $c$ and $u_{a}$ are large in high pressure side of a compression region compared to the low pressure region in front of the wave.
} 


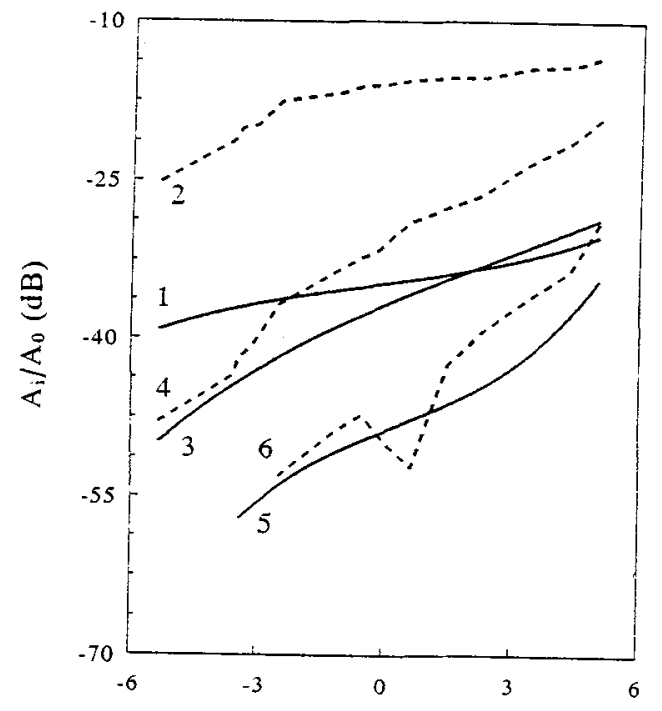

$A_{0}(d B)$

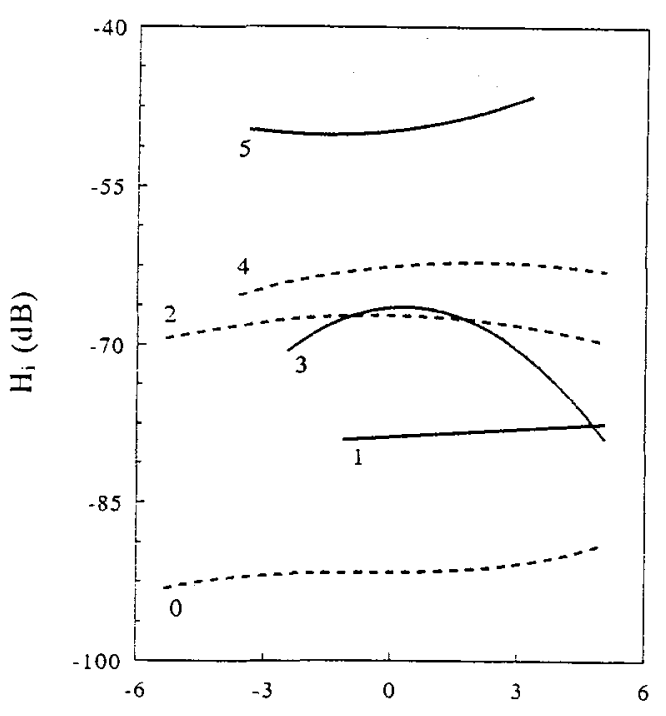

$A_{0}(d B)$

Figure 2: Dependence of the amplitudes $\left(A_{i}\right)$ of the harmonics in the barrel pressure (measured $3 \mathrm{~cm}$ downstream of the mouthpiece) and the transfer function $H_{i}=\left(A_{i}\right)_{e x t} /\left(A_{i}\right)$ from the barrel to a listener at $2 \mathrm{~m}$ from the instrument, on the amplitude $\left(A_{0}\right)$. The D3 note, $f_{0}=148.5 \mathrm{~Hz}$, is the lowest note of the instrument. The reference pressure $p_{\text {ref }}$ used in the $\mathrm{dB}$ scale is $3160 \mathrm{~Pa}$.

starts forming. As explained by Backus and Hundley[42] and by Disselhorst and van Wijngaarden[32] in a resonator pipe closed at both ends, this non-linear effect accumulates while the wave propagates up and down the pipe. A shock is reached even at low amplitudes if frictional wave attenuation is sufficiently low. In contrast the open pipe end of the clarinet implies an inversion of sign of nonlinear effects each time the wave reflects at the open end. Therefore the relevant length $x$ in a wind instrument is the pipe length $L_{p}$ rather than a frictional attenuation distance ${ }^{13}$. At fortissimo levels $A_{0} / p_{a} \approx \Delta p_{m} / 2 p_{a}$ reaches values of $5 \%$. For the lowest register we have: $L_{p} \approx c_{0} / 4 f_{0}$ and the shock formation length is given by: $x_{s}=0.74 L_{p}\left(p_{a} / A_{0}\right)$. This implies that if the waves are harmonic at the mouthpiece, the non-linear wave propagation induces $4 \%$ of first harmonic at the pipe exit ${ }^{14}$. This can be musically interesting.

As the pressure fluctuations in the clarinet mouthpiece are rather harmonic, the analysis of Backus and Hundley[42] based on equation (3) is justified for a clarinet. In a trombone even at forte or mezzo-forte levels, as shown by the data of Elliott and Bowsher[44] and our own data presented in Figure 3, the pressure fluctuations in the mouthpiece of the trombone are strongly non-harmonic. The mouthpiece pressure shows in the lower registers at forte level a series of sharp picked teeth. The relevant accelaration or pressure increase rate, cannot be calculated as done by Backus and Hundley[42] on the basis of the formula (3) derived for a pure harmonic signal. For the lower registers, the pressure rise times are an order of magnitude shorter than the rise time we would have if the fundamental was dominating ${ }^{15}$. The measured mouthpiece pressure shows for the

\footnotetext{
${ }^{13} \mathrm{~A}$ shock wave can only be formed if the acoustical level is sufficient to produce the shock within one pipe length.

${ }^{14} \mathrm{At}$ higher registers the typical amplitudes in the clarinet decrease and we therefore do not expect stronger nonlinearities.

${ }^{15}$ The occurence of peaks is, following Backus and Hundley[42] and Elliott and Bowsher[44], due to the low "average flow resistance" of the lips compared to the pipe impedance at the lowest register. As a consequence the flow is only controlled by the lips in a short period when they are almost closed. In this period the flow resistance through the lips induces an interruption of the flow which induces the sharp negative pressure pick observed in the mouthpiece.
} 


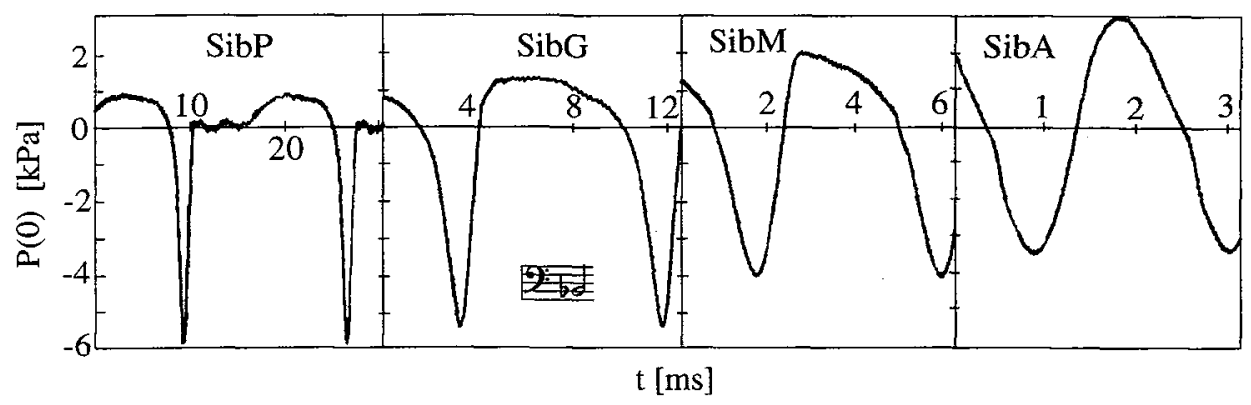

Figure 3: Typical pressures signals at forte levels in the mouthpiece of a bass-trombone.

lowest register such a sharp pressure rise, that we certainly expect that a shock wave formation is possible (Figure 3). For higher registers, the mouthpiece pressure fluctuations in brass instruments is rather harmonic, however the distance the wave travels remains the same as for low registers and the pressure rise time is rather independent of the register. Hence we expect also a significant non-linearity in the wave propagation at higher registers. As our conclusion contradicts the work of Backus and Hundley[42] it is important to note that Peters[45] did obtain schlieren pictures of shock waves formed at the open end of a quarter wave length resonator for pressure rise rate comparable to those measured in brass instruments at fortissimo levels. A further analysis should confirm whether wave propagation non-linearity can explain the non-linearities observed in the transfer function of brass instruments by Beauchamp[41].

7. Conclusions The musical aero-acoustics of the clarinet (volume flow control by the reed, the Bernoulli force on the reed, turbulence, vortex shedding and non-linear wave propagation) is a challenging field of research seeking for more quantitative work. However, it should be obvious that the presently used models described in the literature[7] are excellent first approximations. They provide a basis for time domain simulation which cannot be improved by qualitative aero-acoustical considerations. This conclusion is partially the result of a particular design of the clarinet and is not valid for brass instruments.

The study of the fluid dynamics of the clarinet mouthpiece and the lips of brass players seems to be the most urgent of the aspects discussed above. Quasi-stationary fluid dynamic models of separation as developed by Pelorson[11] can be useful. However, we have some hints that the use of steady flow experiments or stationary flow (numerical) simulations may introduce spurious effects like an exageration of the Coanda effect. Apparently unsteady flow modelling is necessary. Finally the non-linearity of the wave propagation is modest in the clarinet but for brass instruments a more accurate analysis should be carried out before we can draw a definitive conclusion. We expect that this non-linearity contributes to the brightness of brass sound at fortissimo levels. It would certainly be funny if we could confirm that this non-linearity is strong enough to produce shock-waves in trombones.

During the rest of the oscillation period the flow is rather constant and the mouthpiece pressure is close to the player's mouth pressure $p_{m}$. It is obvious that if this explanation is correct, the relevant "lip resistance" is strongly affected by the lip motion and a quasi-stationary approximation ignoring the air displacement by the lips can be very inaccurate. In particular, in analogy with the glottis, abrupt transitions can be expected in the final part of the closing phase of the lip motion. The Bernoulli force and related boundary layer separation phenomena are expected to be crucial in this phase. Using lip models with two mechanical degrees of freedom as proposed by Strong[47] and Titze[46] in combination with the fluid dynamics of Pelorson[11] might provide some insight. 


\section{REFERENCES}

[1] Bouasse H., Tourbillons(Vol.II),Bib. scien. de l'Ingenieur et du Physicien (1936).

[2] RESONANS: software for wind-instrument design, developed at LAUM(Le Mans) in collaboration with the IRCAM(Paris), distributed by CTTM(Le Mans).

[3] McIntyre M.E.,Schumacher R.T. and Woodhouse J., J.Acoust.Soc.Am. 74(1983) 1325-1345.

[4] Ducasse E., Sup.J.Phys., Colloque de Physique 51 (1990) C2-873-876.

[5] Fabre B., La production du son dans les instruments de musique a embouchure de flûte, $\mathrm{PhD}$ thesis,Lab.d'acoustique Musicale, Univ. Paris VI and Univ. du Maine, Le Mans (1992).

[6] Mahu W.E.A., Peters M.C.A.M. Peters, Verge M.-P., Wijnands A.P.J., Fabre B. and Hirschberg A., Attack transient of a flue organ pipe", in Topics in Applied Mechanics, Dijksman J.F. and Nieuwstadt F.T.M. (eds), Kluwer Academic Pub., The Netherlands (1993) pp. 163-171.

[7] Flectcher N.H. and Rossing T.D., The physics of musical instruments, (Springer-Verlag, NY, 1991).

[8] Howe M.S., J.Fluid Mech. 71 (1975) 625-673.

[9] Fletcher N.H., Ann. Rev. Fluid Mech. 11 (1979) 123-146.

[10] Ishizaka K. and Matsudaira M., Fluid Mechanical Considerations of Vocal Cord Vibration,(SCRL Monograph $N^{0} 8$, Speech Com. Research Lab., Inc., S.B.,CA. 1972) .

[11] Pelorson X., Hirschberg A., van Hassel R.R., Wijnands A.P.J. and Auregan Y., Theoretical and experimental study of quasi-steady flow separation within the glottis during phonation. Application to a modified two-mass model, (Manuscript $N^{0}$ 952, Institute for Perception Research, Eindhoven 1993).

[12] Hirschberg A., van de Laar R.W.A., Marrou-Maurieres J.P., Wijnands A.P.J., Dane J.H., Kruijswijk S.G. and Houtsma A.J.M., Acustica 70 (1990) 146-154.

[13] van Zon J., Hirschberg A., Gilbert J. and Wijnands A.P.J., J.Phys., Colloque de Physique Sup. 2, 51(1990) C2-821-824.

[14] Hirschberg A., Gilbert J., Wijnands A.P.J. and Houtsma A.J.M., NAG Journal (Nederlands Akoestisch Genootshap) 107 (1991) 31-43.

[15] Maurin L.," Confrontation théorie-expérience des grandeurs d'entrée d'un excitateur à anche simple" DEA thesis, LAUM, Le Mans (1992).

[16] Valkering A.M.C.,"Characterization of a clarinet mouthpiece", Report $N^{0}$ R-1219-S, Vakgroep Transportfysica, TUE, Eindhoven (1993).

[17] Gilbert J.,"Etude des instruments de musique a anche simple", PhD thesis, LAUM, Le Mans (1991).

[18] Meynial X.,"Systemes micro-intervalles pour instruments a vents a trous lateraux, oscillation d'une anche simple couplee a un resonateur de forme simple", $\mathrm{PhD}$ thesis, LAUM, Le Mans (1987).

[19] Hirschberg A.,(ICP/INPG) Bulletin de la Communication Parlée, 2 (1992) 7-30.

[20] Titze I.R., J.Acous. Soc. Am. 83 (1988) 1536-1552. 
[21] St-Hilaire A.O., Wilson T.A. and Beavers G.S., J.Fluid Mech. 49 (1971) 803-816.

[22] Benade A.H., Fundamentals of musical acoustics,(Oxford Univ. Press, NY 1976, reprint Dover Ed. 1992).

[23] Stevens K.N.,J.Acous.Soc.Am. 50 (1971) 1180-1192.

[24] Rocchesso D. and Turra F., A generalized excitator for real-time sound synthesis by physical modes, paper presented at SMAC 93, Stockholm 1993.

[25] Chafe C., abstract 4aMU3, J.Acous.Soc.Am. 94 Pt2 (1993) 1833.

[26] Schumacher R.T., J.Acous.Soc.Am. 91 (1992) 438-451.

[27] Gibiat V., J.Sound and Vibration 123 (1988) 529-536.

[28] Richard G.,d'Alessandro C. and Grau S.,"Musical noise synthesis using random wavelets", paper presented at Stockholm Musical Acoustics Conference, Stockholm (1993).

[29] Ingard U. and Ising H., J.Acous.Soc. Am. 42 (1967) 6-17.

[30] Anderson A.B.C., J.Acous.Soc.Am. 27 (1955) 1048-1053.

[31] Cummings A., J.Acous.Soc.Am. 79 (1986) 942-951.

[32] Disselhorst J.H.M. and van Wijngaarden L., J.Fluid Mech. 99 (1980) 293-319.

[33] Cummings A. and Eversman W. J.Sound and Vibration 91 (1983) 503-518.

[34] Peters M.C.A.M. and Hirschberg A., J.Sound and Vibration 161 (1993) 281-299.

[35] Peters M.C.A.M., Hirschberg A., Reijnen A.J. and Wijnands A.P.J., J.Fluid Mech. 256 (1993) 499-535.

[36] Wilson J.A., Beavers G.S., De Coster M.A., Holger D.K. and Regenfuss, J.Acous.Soc.Am. 50 (1971) 366-372.

[37] Powell A., ARC 14726 FM 1694 (1951).

[38] Hirschberg A., Bruggeman J.C., Wijnands A.P.J. and Smits N., Acustica 68 (1989) 157-160.

[39] Keefe D.H., J.Acous.Soc.Am. 73 (1983) 1804-1820.

[40] van Wijngaarden L. and Wormgoor J.W., Investigations on resonant acoustic waves in open pipes, in Finite amplitude wave effects in fluid, ed Brorno (1973).

[41] Beauchamp J.W.,"Analysis of simultaneous mouthpiece and output waveforms of wind instruments", paper presented at the 66th meeting of the Audio Engineering Society, L.A.(1980).

[42] Backus J. and Hundley T.C., J.Acous.Soc.Am. 49 (1971) 509-519.

[43] Pierce A.D., Acoustics, (2 ed.,Acous.Soc.Am.,NY 1989).

[44] Elliott S.J. and Bowsher J.M., Journal of Sound and Vibration 83 (1982) 181-217.

[45] Peters M.C.A.M.,"Aeroacoustical sources in internal flows", PhD thesis, T.U. Eindhoven (1993).

[46] Titze I.R., "From tenor voice to trumpet", paper presented at the Stockholm Musical Acoustics Conference (1993).

[47] Strong W.J., Dudley D.J. and Copley D., "Simulation of a player-trumpet system", paper presented at the Stockholm Musical Acoustics Conference (1993). 